\title{
QUESTION OF GLOBAL ASYMPTOTIC STABILITY IN STATE-VARYING NONLINEAR SYSTEMS
}

\author{
MAU-HSIANG SHIH AND JINN-WEN WU \\ (Communicated by Charles Pugh)
}

\begin{abstract}
A problem raised by LaSalle (i.e., a discrete counterpart of the Jacobian problem in differential equations) concerning the global asymptotic stability in state-varying nonlinear systems is settled. A global asymptotic stability criterion for state-varying systems based on vector energy functions is introduced.
\end{abstract}

Let $R^{m}$ denote the $m$-dimensional real vector space of all column vectors and $R^{m \times m}$ the vector space of all real $m \times m$ matrices. For $A \in R^{m \times m}$, let $r(A)$ be the spectral radius of $A$. Consider the state-varying nonlinear system

$$
x^{\prime}=A(x) x,
$$

where $x, x^{\prime}$ are functions on $\{0,1, \ldots\}$ to $R^{m}$ related by the formula

$$
x^{\prime}(k)=x(k+1), \quad k=0,1, \ldots,
$$

and $A: R^{m} \rightarrow R^{m \times m}$ is continuous. In his book [1, p. 21], LaSalle asked: "if $r\left(A(x)<1\right.$ for each $x \in R^{m}$, is the origin globally asymptotically stable for (1)?". LaSalle's problem is of importance, because it is a discrete counterpart of the famous Jacobian problem in differential equations [2]. The answer of LaSalle's problem is negative, as the following example shows.

Define $A: R^{2} \rightarrow R^{2 \times 2}$ by

$$
\begin{array}{ll}
A(x)=\left(\begin{array}{ll}
0 & 2 \\
0 & 0
\end{array}\right) & \text { if } x_{2}-x_{1} \geq 1, \\
A(x)=\left(\begin{array}{ll}
0 & 2\left(x_{2}-x_{1}\right) \\
0 & 0
\end{array}\right) & \text { if } 0 \leq x_{2}-x_{1} \leq 1, \\
A(x)=\left(\begin{array}{cc}
0 & 0 \\
2\left(x_{1}-x_{2}\right) & 0
\end{array}\right) & \text { if }-1 \leq x_{2}-x_{1} \leq 0, \\
A(x)=\left(\begin{array}{ll}
0 & 0 \\
2 & 0
\end{array}\right) & \text { if } x_{2}-x_{1} \leq-1 .
\end{array}
$$

Received by the editors January 11, 1992 and, in revised form, February 22, 1993.

1991 Mathematics Subject Classification. Primary 39A11, 39B12; Secondary 58F10.

Key words and phrases. Global asymptotic stability, LaSalle's problem, state-varying system, spectral radius. 
Then for each $x \in R^{2}$, we have $r(A(x))=0$. Set $x(0)=\left(\begin{array}{l}0 \\ 1\end{array}\right)$. Then

$$
x(2 k)=\left(\begin{array}{c}
0 \\
2^{2 k}
\end{array}\right), \quad k=0,1, \ldots
$$

Thus the origin is not a global attractor for (1). This completes our solution of LaSalle's problem.

This example also indicates that even the following stronger spectral radius condition and norm condition: there exist constants $\alpha>0$ and $\beta>0$ such that

$$
\|A(x)\| \leq \alpha \text { for all } x \in R^{m} \quad \text { and } \quad r(|A(x)|) \leq \beta<1 \quad \text { for all } x \in R^{m},
$$

where $|A(x)|$ denotes the absolute value of $A(x)$, do not suffice for the global asymptotic stability of the origin in (1). Therefore, seeking a different approach to determine the global asymptotic stability of the origin in (1) might be significant. We shall be concerned with giving a global asymptotic stability criterion for (1) based on a combination of the vector energy functions and the comparison principle.

Suppose that for each $x \in R^{m}, A(x)$ in (1) is partitioned in the manner

$$
\left(A_{i j}(x)\right)_{1 \leq i, j \leq s}, \quad \text { where } A_{i j}: R^{m} \rightarrow R^{m_{i} \times m_{j}}, \sum_{l=1}^{s} m_{l}=m .
$$

Suppose that for each $i=1, \ldots, s$, there exists a norm $n_{i}$ on $R^{m_{i}}$ such that

$$
\sup _{x \in R^{m}} \sup _{n_{j}(y)=1} n_{i}\left(\left(A_{i j}(x)\right) y\right) \leq \alpha_{i j}<+\infty \quad(j=1, \ldots, s) .
$$

Let $C=\left(\alpha_{i j}\right) \in R^{s \times s}$.

Theorem. If $r(C)<1$, then there exist a norm $\|\cdot\|$ on $R^{m}$ and constants $\kappa>0$ and $0<\mu<1$ such that the solution of (1) satisfies

$$
\|x(k)\| \leq \kappa \mu^{k}\|x(0)\|, \quad k=1,2, \ldots .
$$

Consequently, the origin is globally exponentially stable for (1).

Proof. Define the vector energy function

$$
V(x)=\left(\begin{array}{c}
n_{1}\left(x^{1}\right) \\
\vdots \\
n_{s}\left(x^{s}\right)
\end{array}\right), \quad x^{i} \in R^{m_{i}}(i=1, \ldots, s) .
$$

Then for $k=0,1, \ldots$,

$$
\begin{aligned}
V(x(k+1)) & =\left(\begin{array}{c}
n_{1}\left(x^{1}(k+1)\right) \\
\vdots \\
n_{s}\left(x^{s}(k+1)\right)
\end{array}\right)=\left(\begin{array}{c}
n_{1}\left(\sum_{j=1}^{s} A_{1 j}(x(k)) x^{j}(k)\right) \\
\vdots \\
n_{s}\left(\sum_{j=1}^{s} A_{s j}(x(k)) x^{j}(k)\right)
\end{array}\right) \\
& \leq\left(\begin{array}{c}
\sum_{j=1}^{s} \alpha_{1 j} n_{1}\left(x^{j}(k)\right) \\
\vdots \\
\sum_{j=1}^{s} \alpha_{s j} n_{s}\left(x^{j}(k)\right)
\end{array}\right)=C V(x(k)),
\end{aligned}
$$


where the notation that one vector is less than or equal to another refers to the condition that each component of the first vector is less than or equal to the corresponding component of the second vector. By the spectral radius theorem it follows easily that

$$
\left\|x(k) \leq \kappa \mu^{k}\right\| x(0) \|, \quad k=1,2, \ldots,
$$

where $r(C)<\mu<1, \kappa$ is a constant, and the norm $\|\cdot\|$ is given by $\|x\|=$ $\sum_{i=1}^{s} n_{i}\left(x_{i}\right)$. This completes the proof of the theorem.

As an illustration, we consider the four-dimensional state-varying discrete system:

$$
\begin{aligned}
& x(k+1)=A_{1}\left(\begin{array}{l}
x(k) \\
y(k)
\end{array}\right) x(k)+A_{2}\left(\begin{array}{l}
x(k) \\
y(k)
\end{array}\right) y(k), \\
& y(k+1)=A_{3}\left(\begin{array}{l}
x(k) \\
y(k)
\end{array}\right) x(k)+A_{4}\left(\begin{array}{l}
x(k) \\
y(k)
\end{array}\right) y(k),
\end{aligned}
$$

where $x(k), y(k) \in R^{2}$ and $A_{i}: R^{4} \rightarrow R^{2 \times 2} \quad(1 \leq i \leq 4)$ are given by

$$
\begin{aligned}
& A_{1}\left(\begin{array}{l}
x \\
y
\end{array}\right)=\left(\begin{array}{cc}
0.8 \cos ^{2}\left(y_{1}\right) & 0.8 \sin ^{2}\left(y_{1}\right) \\
0.8 \sin ^{2}\left(y_{2}\right) & 0.8 \cos ^{2}\left(y_{2}\right)
\end{array}\right), \\
& A_{2}\left(\begin{array}{l}
x \\
y
\end{array}\right)=\left(\begin{array}{cc}
0.1 x_{1} /\left(1+\|x\|_{2}\right) & 0 \\
0 & 0.1 x_{2} /\left(1+\|x\|_{2}\right)
\end{array}\right), \\
& A_{3}\left(\begin{array}{l}
x \\
y
\end{array}\right)=\left(\begin{array}{cc}
0.2 y_{1} /\left(1+\|y\|_{2}\right) & 0 \\
0 & 0.2 y_{2} /\left(1+\|y\|_{2}\right)
\end{array}\right), \\
& A_{4}\left(\begin{array}{l}
x \\
y
\end{array}\right)=\left(\begin{array}{cc}
0.8 \cos ^{2}\left(x_{1}\right) & 0.8 \sin ^{2}\left(x_{2}\right) \\
0.8 \sin ^{2}\left(x_{1}\right) & 0.8 \cos ^{2}\left(x_{2}\right)
\end{array}\right) .
\end{aligned}
$$

Then, for all $\left(\begin{array}{l}x \\ y\end{array}\right) \in R^{4}$, we have

$$
\begin{gathered}
\left\|A_{1}\left(\begin{array}{l}
x \\
y
\end{array}\right)\right\|_{\infty}=0.8, \quad\left\|A_{4}\left(\begin{array}{l}
x \\
y
\end{array}\right)\right\|_{1}=0.8, \\
\sup _{\|w\|_{1}=1}\left\|A_{2}\left(\begin{array}{l}
x \\
y
\end{array}\right) w\right\|_{\infty} \leq 0.1, \quad \sup _{\|w\|_{\infty}=1}\left\|A_{3}\left(\begin{array}{l}
x \\
y
\end{array}\right) w\right\|_{1} \leq 0.2 .
\end{gathered}
$$

Let $C=\left(\begin{array}{ll}0.8 & 0.1 \\ 0.2 & 0.8\end{array}\right)$. Then $r(C)<1$, and hence the theorem may now be applied to conclude that the origin is globally asymptotically stable for (2). However, set

$$
D\left(\begin{array}{l}
x \\
y
\end{array}\right)=\left(\begin{array}{cc}
A_{1}\left(\begin{array}{l}
x \\
y
\end{array}\right) & A_{2}\left(\begin{array}{l}
x \\
y
\end{array}\right) \\
A_{3}\left(\begin{array}{l}
x \\
y
\end{array}\right) & A_{4}\left(\begin{array}{l}
x \\
y
\end{array}\right)
\end{array}\right)
$$

then, for all $\left(\begin{array}{l}x \\ y\end{array}\right) \in R^{4}$, we have

$$
\left|D\left(\begin{array}{l}
x \\
y
\end{array}\right)\right| \leq M=\left(\begin{array}{cccc}
0.8 & 0.8 & 0.1 & 0 \\
0.8 & 0.8 & 0 & 0.1 \\
0.2 & 0 & 0.8 & 0.8 \\
0 & 0.2 & 0.8 & 0.8
\end{array}\right)
$$


and $r(M)>1$. Therefore, the usual comparison result is not applicable to conclude that the global asymptotic stability of the origin in (2).

\section{ACKNOWLEDGMENT}

The authors express their deep appreciation to Professor Pugh for valuable comments.

\section{REFERENCES}

1. J. P. LaSalle, The stability of dynamical systems, CBMS-NSF Regional Conf. Ser. in Appl. Math., vol. 25, SIAM, Philadelphia, PA, 1976.

2. G. H. Meisters, Jacobian problems in differential equations and algebraic geometry, Rocky Mountain J. Math. 12 (1982), 679-705.

Department of Mathematics, Chung Yuan University, Chung-Li, Taiwan 320

E-mail address: mhshihecauchy. cycu.edu.tw 